Mitteilungen der Österreichischen Geographischen Gesellschaft,

156. Jg. (Jahresband), Wien 2014, S. 171-190

\title{
Historische W ALDNUTZUNG UND GEOMORPHOLOGISCHE Folgewirkungen im Einzugsgebiet der Eisenproduktion des Steirischen Erzberges
}

\author{
Stefan Premm und Christine Embleton-Hamann, beide Wien* \\ mit 4 Abb. und 2 Tab. im Text
}

\section{INHALT}

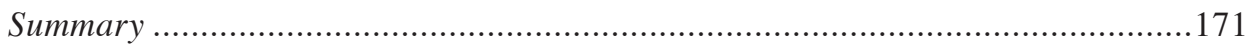

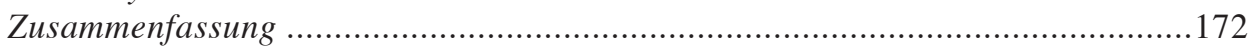

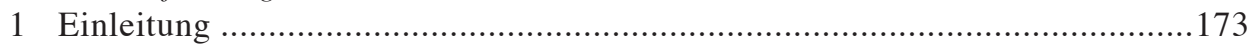

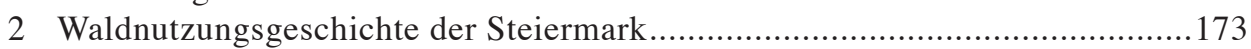

3 Produktion von Holzkohle für die obersteirische Eisenindustrie .....................175

4 Methodische Zugänge zur Erfassung raum-zeitlicher Hotspots

der Waldverwüstung .................................................................. 176

5 Geomorphologische Folgewirkungen von Eingriffen in die Walddecke ..........182

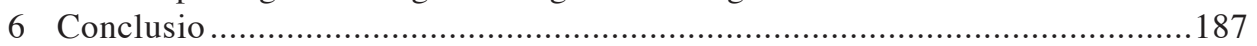

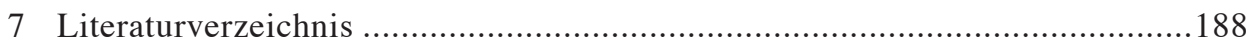

\section{Summary}

Deforestation and its geomorphic impact triggered by historic iron-ore production at the Styrian Erzberg

The forests of the northern Styria region have been exploited since ca. 3,000 BC. Logs and charcoal were used as an energy source to produce salt, copper and iron. Since the early $8^{\text {th }}$ century AD a continuous ore-mining tradition has been documented for the Erzberg. In the middle of the $13^{\text {th }}$ century the iron production increased remarkably, causing an escalating charcoal demand that has continued to increase. Virgin woodland areas were logged primarily for charcoal production. The massive charcoal demand, which was covered by clear cutting of extensive forest areas, ceased abruptly in the late $19^{\text {th }}$ century, when fossil coal was used instead of charcoal.

* Mag. Stefan Premm und ao.Univ.-Prof. Mag. Dr. Christine Embleton-Hamann, beide Universität Wien, Institut für Geographie und Regionalforschung, Universitätsstraße 7, A-1010 Wien; E-Mail: s.premm@ gmx.at; christine.embleton-hamann@univie.ac.at; http://geographie.univie.ac.at Die Autoren möchten einem unbekannten Gutachter für wertvolle Anregungen zur Verbesserung des Manuskripts danken. 
The first goal of the paper is to identify methodological approaches for the spatiotemporal reconstruction of hotspots of deforestation within the Erzberg region from historical documents. To focus on forest management practice and log transportation structures seems to be critical. In this way historic forest evaluations may allow local reconstruction of the state of the forest. A more regional picture can be gained from records of historical iron-ore production, the charcoal demand of iron manufacture and pollen analysis. Land-use practices in northern Styria had considerable effects on the vegetation cover, the hydrological cycle and geomorphic processes. Some of these effects were already tentatively addressed in the literature of the $19^{\text {th }}$ century.

The second goal of the paper is to highlight the increased sediment mobilisation caused by two of the dominant historical land-use practices, namely slash and burn and extensive clear cutting. In the course of time they were interrupted by natural forest regeneration, another factor that needs to be taken into account. Finally a soil exposure is presented, showing how the increased sediment flux is documented in the associated accumulation areas at the mouth of tributaries or foothill areas.

\section{Zusammenfassung}

Im Gebiet der nördlichen Steiermark wurden die Ressourcen der Waldgebiete seit etwa 3000 v. Chr. intensiver genutz.t. Holz und Holzkohle dienten als einzige Energiequelle zur Herstellung von Salz, Kupfer und Eisen. Seit dem frühen 8. Jh. n. Chr. ist eine kontinuierliche Eisenbergbautradition am Erzberg überliefert. Die Eisenproduktion erfuhr um 1250 einen Industrialisierungsschub, der die ausgestoßenen Eisenmengen sowie den damit verbundenen Holzkohlenverbrauch enorm ansteigen ließ. Der große Holzkohlenbedarf wurde bis zur Einführung von Mineralkohle als Energieträger zu Ende des 19. Jhs. überwiegend durch das Anlegen von Großkahlschlägen abgedeckt.

Methodische Zugänge zur raum-zeitlichen Rekonstruktion von Hotspots der Rodungstätigkeit werden thematisiert sowie deren Verbindung mit den historischen Landnutzungsformen und den Anlagen zur Holzbringung aufgezeigt. Ein Überblick über die historischen Waldbestandsaufnahmen verdeutlicht deren Potenzial für eine lokale Rekonstruktion des Waldzustandes. Als Möglichkeiten zur regionalen Bewertung der Vegetationsdecke werden die Darstellung der historischen Eisenproduktion am Erzberg, Angaben über den Holzkohlenbedarf einzelner Gewerke sowie Informationen aus palynologischen Untersuchungen beschrieben.

Die historische Waldnutzung im Erzberggebiet hatte signifikante Auswirkungen auf die Vegetationsbedeckung und somit auf geomorphologische Prozesse an den genutzten Hängen. Darauf deuten bereits historische Einträge aus dem 19. Jh. hin. Die generellen Auswirkungen zweier in der nördlichen Steiermark nahezu flächendeckend praktizierter Landnutzungsformen - Brandwirtschaft und Großkahlschläge - sowie die Folgewirkungen einer Regeneration von genutzten Waldungen werden dargestellt. Abschließend wird anhand eines Bodenaufschlusses exemplarisch aufgezeigt, wie sich die verstärkte Hangabtragung in den Ablagerungsbereichen am Talausgang dokumentiert. 


\section{Einleitung}

Aus der älteren Literatur zur Montanindustrie am Steirischen Erzberg (MENHARDT 1754-1762; Sснцiск et al. 1767; Sснорғ \& Schof 1843, u.a.) geht klar hervor, dass die Eisenproduktion über Jahrhunderte zu einem Raubbau an den Wäldern des gesamten steirischen Alpenraums führte, der in seinem Ausmaß und in seiner Schonungslosigkeit überraschend und mit der heutigen Rodungstätigkeit in den Schwellen- und Entwicklungsländern durchaus vergleichbar ist. Auch in den außereuropäischen Industriestaaten war ein solcher Raubbau bis gegen Ende des 20. Jhs. verbreitet. In Europa aber, wo sich der Nachhaltigkeitsgedanke gerade auf dem Boden der Forstwirtschaft entwickelte, gibt es kein kollektives Gedächtnis und wenig Fachliteratur zum wahren Ausmaß der ehemaligen Waldverwüstungen.

Für Geomorphologen steht außer Frage, dass massive Waldrodung zu Bodenerosion, Rutschungen und Murabgängen führen muss, das heißt zu einer insgesamt stark erhöhten Abtragung und Sedimentmobilisierung. Die Frage der Auswirkungen der historischen Eisenproduktion am Erzberg ist daher für zwei aktuelle Forschungsthemen der Geomorphologie äußerst interessant, nämlich für die Sedimentbilanzierung und für die Anthropogeomorphologie. Bislang gibt es zu diesen beiden Forschungsthemen keine einschlägigen Untersuchungen aus dem steirischen Alpenraum. Dazu müsste zuerst geklärt werden, welche Täler zu welchem Zeitpunkt von den Rodungen betroffen waren und welche historischen Wirtschaftsformen dabei zum Einsatz kamen.

Ziel der vorliegenden Arbeit ist erstens die Entwicklung von methodischen Zugängen, mit denen die raum-zeitlichen Hotspots der historischen Waldverwüstungen für die Eisenproduktion am Erzberg identifiziert werden können. Zweitens werden die damit verbundenen Landnutzungsformen, nämlich Brandwirtschaft, Kahlschlagbetrieb und natürliche Wiederbewaldung auf ihre geomorphologischen Folgewirkungen untersucht. Die Arbeit ist ein Workshop-Bericht aus einem laufenden Dissertationsprojekt. Daher mussten sich die Autoren bei der Veranschaulichung der geomorphologischen Folgewirkungen auf themenrelevante, bereits veröffentlichte Literaturbeispiele beschränken.

\section{Waldnutzungsgeschichte der Steiermark}

In der Steiermark erfolgten die ersten größeren Eingriffe in die Urwälder bereits ab etwa 3000 v. Chr. Die Illyrer betrieben Salzbergbau in jenem Gebiet, in dem sich um 800 v. Chr. die Hallstattkultur entwickelt hat (Pömer \& Straub 1980). Neben dem Salzbergbau wurden, ebenfalls in ur- und frühgeschichtlicher Zeit, Erzabbau und Metallverhüttung in den steirischen Bergen betrieben. Spätestens seit der Mittleren Bronzezeit wurde in den Eisenerzer Alpen Kupfererz bergmännisch gewonnen und verhüttet (Drescher-Schneider 2003; Klemm 2003). Mit dem Eindringen der Kelten, etwa um 400 v. Chr., erhöhte sich die bergbauliche Aktivität in der Obersteiermark. Der zusätzliche Bevölkerungsdruck und der vermehrte Bergbau sorgten für eine weitere Auflichtung der Wälder (HAFNER 1979). 
Bereits zur Römerzeit wurde das „norische Eisen“ vom Erzberg gerühmt und zur Herstellung von Waffen verwendet. Mit dem Ende der römischen Herrschaft und dem Beginn der Völkerwanderungen verloren sich die Berichte über die steirische Eisengewinnung. Erst um das Jahr 712 wird wieder vom Erzbergbau am Erzberg berichtet (HAFNER 1979). Die historischen Nachweise sind zwar recht dürftig, dennoch muss man annehmen, dass die intensive Besiedlung im Bereich des heutigen Eisenerz im 9., spätestens aber im 10. Jh. eingesetzt hat (DRESCHER-SCHNEIDER 2003). Seither belegen historische Quellen eine kontinuierliche Bergbautradition und Siedlungsgeschichte am und um den Steirischen Erzberg (KLEmm 2003, S. 25).

Zu dieser Zeit wurde das Erz größtenteils mit einfachsten bergmännischen Mitteln direkt an der Oberfläche gewonnen. Diese Methode ist in einer Darstellung aus dem Jahr 1557 in Abbildung 1 links zu sehen (AgRICOLA 1557).
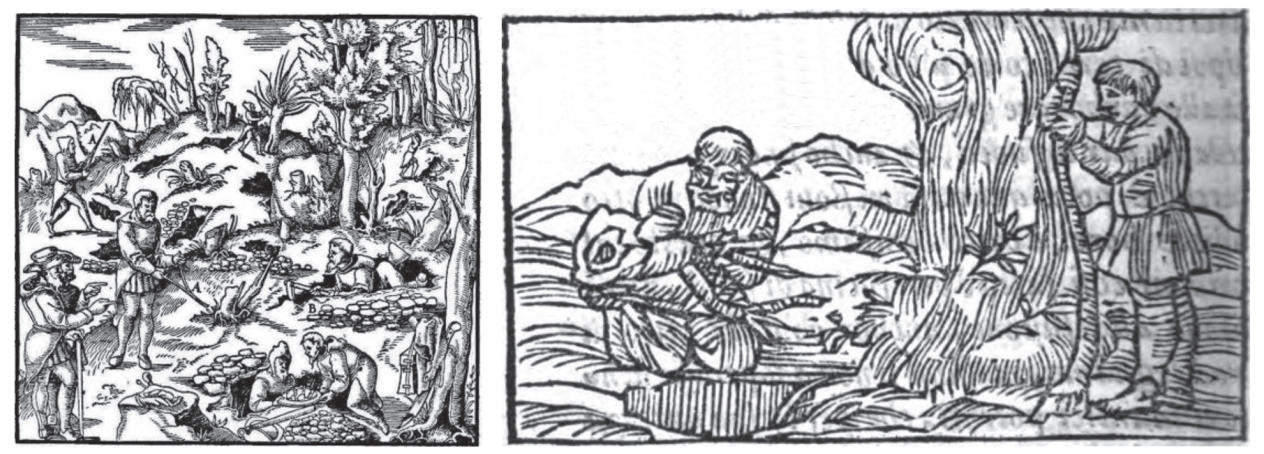

Abb. 1: Links: Früheste Formen der Eisenerzgewinnung (Agricola 1557). Rechts: Bildliche Darstellung der Grubenverkohlung (BIRINGUCCIO 1559)

Geschmolzen wurde teils am Erzberg selbst und teils am Präbichl in kleinen, an windseitigen Berghängen gelegenen Gruben. Die Verkohlung fand ebenfalls in Gruben statt wie es in einer Zeichnung aus dem 16. Jh. in Abbildung 1 rechts festgehalten wurde (BIRINGUCCIO 1559). Die Bearbeitung in den Ortschaften Innerberg (dem heutigen Eisenerz) und Vordernberg erfolgte erst, als durch neue Techniken und Ausweitungen der Fördermengen mehr Raum benötigt wurde (HAFNER 1979). Um 1250 ging man von mittelalterlichen Gewinnungsverfahren zum Stuckofen oder Stückofen über, der bereits in Hütten errichtet wurde. Zum Antrieb der stärkeren Gebläse des Stuckofens mittels Wasserkraft errichtete man große Wasserräder, weshalb die Eisenschmelzhütten Innerbergs und Vordernbergs seit dieser Zeit Radwerke bzw. wegen der starken Gebläse Blaahaus oder Plähaus genannt wurden. Diese Umstellung Mitte des 13. Jhs. markiert den Beginn der industriellen Eisengewinnung, der Ausstoß an Roheisen wurde erheblich gesteigert (HAFner 1979; Mayrhofer \& Hampl 1958). Bis zum 19. Jh. folgten dann Weiterentwicklungen in der Montanindustrie und der Forstwirtschaft, die immer höhere Produktionszahlen ermöglichten und dem Wald immer mehr Rohstoffe abverlangten.

Am Beginn und zur Mitte des 19. Jhs. war der Bedarf an Holzkohle ungebrochen. Im k.k. Ackerbauministerium sind für das Jahr 1895 in der Steiermark noch immer 115 ständige Meilerköhlereien, 280 stehende Meiler und 158 liegende Meiler verzeichnet 
(HafNer 1979, S. 157). Nach der Erschließung des Landes durch die Eisenbahn kam Holzkohle auch aus anderen Teilen der Donaumonarchie, wodurch sich der Druck auf die obersteirischen Wälder verringerte. Neue Verhüttungstechniken zu Ende des 19. Jhs. ermöglichten den Einsatz von Koks und mineralischer Kohle als Energieträger. Der Bedarf an Holzkohle endete schließlich abrupt, 1903 wurden alle Holzkohlenlieferungen zum Erzberg eingestellt. Die Wälder wurden fortan in Nutzholzwirtschaft geführt (HAFNER 1979).

\section{Produktion von Holzkohle für die obersteirische Eisenindustrie}

Mit den Produktionssteigerungen, die zum Beginn der Neuzeit eintraten, erhöhte sich auch die Bevölkerungszahl rund um den Erzberg. Neue Freiflächen für die Besiedlung, zur Viehweide und für den Ackerbau mussten geschaffen werden. Doch den weitaus größten Druck auf die Waldressourcen übte der enorme und ständig steigende Holzkohlenbedarf aus. Holzkohle in ausreichender Menge war unerlässlich um erstens die zum Schmelzen des Eisens nötigen hohen Temperaturen zu erreichen und um zweitens durch den reinigenden Kohlenstoff in der Holzkohle das Endprodukt erheblich zu verbessern (Ast et al. 1970; HaFNER 1979; Killian 1994).

Die genutzten Waldgebiete reichten bald nicht mehr aus. Im Lauf der Jahrhunderte wurden immer wieder Möglichkeiten gesucht, neue, entlegenere Waldungen aufzuschließen und nutzbar zu machen. Durch die Trennung des Ortes der Rohstoffentnahme und des Ortes der Verkohlung gelang dies auf eine äußerst effiziente Weise. Das Holz wurde an Hängen in waldreichen Einzugsgebieten geschlagen und mittels Riesen zu den Flussläufen ins Tal befördert. Abbildung 2 links zeigt eine Photographie der Riesanlage im Bruckgraben im Gesäuse. Der weitere Transport erfolgte auf dem Wasserweg durch Eigenwassertrift, vor allem zurzeit der Schneeschmelze. Reichte das Wasser in den Flüssen nicht aus, wurden Klausanlagen errichtet, welche nach Öffnung der Klaustore die Trift auf Schwallwasser ermöglichten. Eine der bekanntesten Klausanlagen der
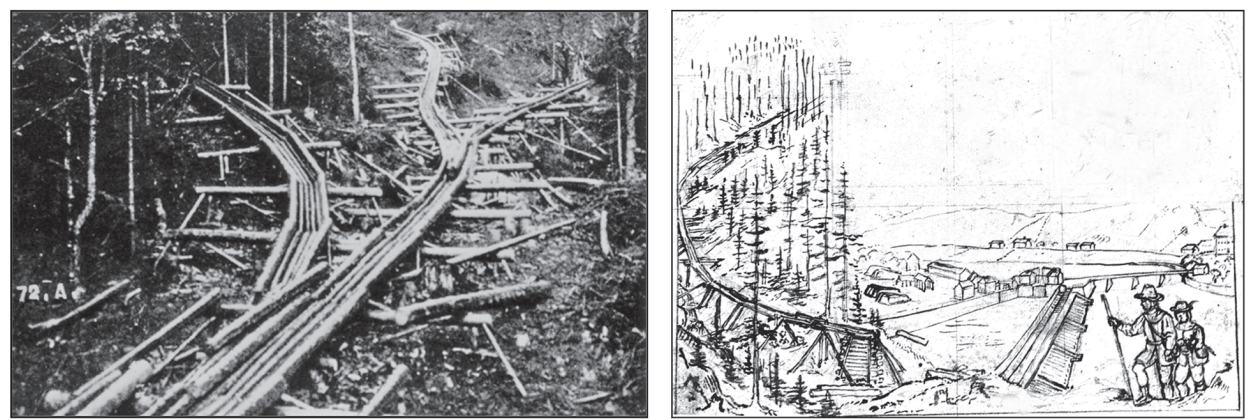

Abb. 2: Links: Holzriesen im Bruckgraben, Gesäuse (HasitschKa 2005). Rechts: Symbolhafte Darstellung von Riese und Rechen in Hieflau aus dem Jahr 1790 (HASITSCHKA 2005) 
Obersteiermark, die Prescenyklause, befindet sich in der Nähe von Weichselboden, zwischen Wildalpen und Gußwerk. Aufgefangen wurden die Baumstämme mit quer zum Flusslauf erbauten Holzrechen, die sich an zentralen Verkohlungsorten befanden. Hier wurde das Kohlholz gelagert und getrocknet, sodass ein ganzjähriger Betrieb der Kohlenmeiler gewährleistet war. In der Abbildung 2 rechts aus dem Jahr 1790 ist der Bringungsweg von der Riese zum Holzrechen Hieflau schematisch dargestellt (HAFNER 1979; HASITSCHKA 2005).

Für die Holzkohlenproduktion war ausschließlich die Menge an Holz entscheidend. Beschädigungen und Verluste von bis zu 25\% während der Trift nahm man billigend in Kauf, war sie doch die einzige Möglichkeit, die zahlreichen Kohlenmeiler in den Zentralköhlereien zu bedienen.

\section{Methodische Zugänge zur Erfassung raum-zeitlicher Hotspots der Waldverwüstung}

Die Nutzung der Wälder rund um den Erzberg war über Jahrhunderte hinweg äußerst intensiv und betraf fast alle Täler der Obersteiermark. Im Folgenden werden einige methodische Zugänge vorgestellt, die es ermöglichen, das Ausmaß der historischen Waldnutzung auch für einzelne Täler zu rekonstruieren und Hinweise auf den Zustand der Vegetationsbedeckung im Laufe dieser Nutzungsphase zu erhalten.

\subsection{Bedeutung der Rodungsart}

Vor dem Aufschwung der Eisenproduktion zu Beginn der Neuzeit beschränkte sich die Nutzung des Waldes zumeist auf die unmittelbare Umgebung von Ansiedlungen und Almen. Es wurden Bau-, Heiz- und Zaunholz mittels Plenterwirtschaft (Einzelstammentnahme) geschlagen, Waldflächen wurden extensiv beweidet und zur Einstreugewinnung verwendet. Kahlschläge waren selten und wenn sie erfolgten, dann nur sehr kleinflächig, beispielsweise zur Gewinnung einer neuen Weide- oder Ackerfläche.

Mit dem starken Anstieg des Eisenausstoßes veränderte sich die Bewirtschaftungsart der obersteirischen Wälder gründlich. Der Bedarf an Holzkohle war enorm und stieg im gleichen Maße wie die Eisenproduktion. Die Plenterwirtschaft erwies sich schnell als nicht wirtschaftlich und effizient genug, um die großen Mengen an Kohlholz zur Verfügung zu stellen. Die hauptsächliche Nutzungsart veränderte sich in Richtung Großkahlschläge. Diese Bewirtschaftungsart bestimmte somit über Jahrhunderte hinweg das Bild in den Wäldern im Einzugsgebiet des Erzberges. Darüber berichtet unter anderen auch Нlubeк (1846, S. 89f.), der die „... Plenterwirthschaft ... als die natürlichste, einfachste und nachhaltigste Bewirthschaftungsweise in den Alpenländern

..." hervorhebt. Vor allem in höheren Lagen, in Gebieten, wo „Schneelawinen und Erdabsetzungen eintreten“, sowie an steilen Hängen mit nur spärlicher Vegetation und wo ,... die Forstbestände gegen Sturzwinde zu schützen ...“ seien. „Doch ungeachtet der Wichtigkeit der Plenterwirthschaft in diesen Fällen ist dieselbe gegenwärtig in der 
Steiermark auf kleine Parzellen des Rustikalbesitzstandes beschränkt" (HLubeK 1846, S. 90). Als ,... Hauptart der Bewirthschaftung in dem grösseren Theile der Steiermark ...“ beschreibt HцUвEK (1846, S. 90) die Anlegung von Großkahlschlägen folgendermaßen: „Als aber die Montanindustrie an Ausdehnung bedeutend zugenommen hatte, war das Bedürfnis immer fühlbarer, grössere Holzmassen mit einem geringen Kostenaufwande auf einmal zu gewinnen, und es wurde die mit einer beschwerlichen Holzausbringung verbundene Plenterwirthschaft in den kahlen Abtrieb ohne künstlicher Besamung oder Pflanzung verwandelt, durch welchen der nicht erfreuliche Zustand der Wälder in der Steiermark herbeigeführt worden ist.“

Im Laufe der Jahrhunderte kam es dennoch immer wieder zu Engpässen in der Holzversorgung. Die Kahlschläge wurden seit jeher dem natürlichen Anflug aus den Nachbarparzellen überlassen, was eine lange Verjüngungszeit mit sich brachte. Deshalb und wohl auch weil die Kahlschläge immer größere Ausmaße annahmen, wurde es zur üblichen Praxis, einzelne starke Samenbäume bei der Abholzung zu verschonen, die den natürlichen Anflug befördern sollten. Mit dem wachsenden Bewusstsein der Wichtigkeit der Ressourcen des Waldes wurden auch immer größere Anstrengungen unternommen, auf den angelegten Kahlschlägen schnellstmöglich einen gesunden Jungwuchs hervorzubringen. Dies wurde jedoch erst zur Mitte des 19. Jhs. intensiver mit dem Sammeln und Aussäen von Baumsamen sowie durch Aufforstung mittels Setzlingen und Jungpflanzen verfolgt (Ast et al. 1970; Du Hamel du Monceau et al. 1766; Hafner 1979; Hlubek 1844; Hlubek 1846; Leinböck 1834; Nather 1964; WesSELY 1853b, u.a.).

\subsection{Bedeutung von Anlagen zur Holzbringung}

Betrachtet man die Auswirkungen auf die Vegetationsbedeckung, so sind Anlagen zum hangabwärtigen Transport von geschlagenen Baumstämmen, Klausanlagen für den Triftbetrieb sowie Holzrechen bei den Verkohlungsstätten von zentraler Bedeutung. Denn entweder wurden Waldungen in gewissen Gebieten oder Talabschnitten erst durch den Bau von Bringungsanlagen nutzbar gemacht oder die Art der Nutzung veränderte sich nach deren Errichtung zu Großkahlschlägen.

Darauf deutet etwa NATHER (1964) in den Ausführungen über die Wälder des oberen Murtales hin: „Bis zur Mitte des 19. Jahrhunderts war die Kahlschlagwirtschaft die einzige Art der Holzentnahme.“ (NATHER 1964, S. 197) „Um den Riesenbau wirtschaftlich tragbar zu machen, wurden die Kahlschläge möglichst groß gehalten. So haben diese Anlagen (zur Holzbringung) zum Großkahlschlag mit beigetragen.“ (NATHER 1964, S. 184)

Ein weiterer Hinweis auf die Auswirkungen von Bringungsanlagen ist einem Schreiben des k.k. Forstinspektors aus dem September des Jahres 1872 zu entnehmen: „Der Sinn für die Aufforstung der Kahlschläge war bei den meisten Forstgroßbetrieben erst in neuester Zeit zur Tat gediehen. In abgelegenen Gebieten wurde noch Kahlschlag an Kahlschlag gereiht, um die Hauptriesen während ihrer Verwendungsdauer ausnützen zu können.“ (HAFNER 1979, S. 104) Die Anordnung und die Leistungsfähigkeit der Riesanlagen bestimmte damit die Größe des Kahlschlags. Zur Verbesserung 
der Wirtschaftlichkeit strebte man während der Lebensdauer einer Bringungsanlage nach einer konzentrierten Bewirtschaftung der Wälder in ihrem Einzugsgebiet. Man versuchte also mit den Bringungsanlagen in kurzer Zeit möglichst viel Holz ins Tal zu befördern (HAFNER 1979).

In den allermeisten Fällen kam es zur Errichtung von Rechen, Riesen und anderen Bringungsanlagen, um bestimmte Waldgebiete zugänglich und wirtschaftlich nutzbar machen zu können. Ein bekanntes Beispiel dafür ist der Holzrechen in Hieflau. Mit der Fertigstellung des Hieflauer Rechens im Jahr 1516 konnte man die leicht erreichbaren Wälder des oberen Ennstals einer umfassenden Nutzung unterziehen (HAFNER 1979).

Auch in den Wäldern des Gesäuses finden sich Beispiele für die erstmalige Nutzbarmachung von einzelnen Beständen. Im Admonter Archiv findet sich etwa ein Jahr nach Beginn des Rechenbaus in Hieflau der Bericht über die erstmalige Abholzung in den Hochlagen des Hartelsgrabens und in den höchsten Karen unter der Gsuchmauer. Das geschlagene Holz konnte mit Riesen zur Enns gebracht und von dort nach Hieflau zum neu errichteten Rechen getriftet werden (HASITSCHKA 2005).

Auch das Salzatal und dessen Nebentäler konnten erst durch die Errichtung des Großreiflinger Holzrechens im Jahr 1570 nutzbar gemacht werden. Von den Wäldern an der Salza erwarteten die Eisengewerke für „etliche hundert jar Kohlholtz.“ (HAFNER 1979, S. 111)

\subsection{Auswertung historischer Waldbegehungen und -beschreibungen}

Um den ständig befürchteten Holznöten zu begegnen, wurden im Laufe der Jahrhunderte viele Waldbeschreibungen (,Waldbereitungen“, „Waldbeschaue“, „,Waldinventuren") erstellt. Sie wurden von den Besitzern der Eisenindustrie und der Waldungen - das waren zumeist die jeweiligen weltlichen Landesherren - in Auftrag gegeben. Unter anderem wollte man damit den Waldbestand und die sich daraus ergebende Versorgungslage darstellen, Waldverwüstungen aufzeigen und Maßnahmen zu deren Beseitigung vorschlagen. Die Waldbereitungen bildeten deshalb auch die Grundlage für die Waldordnungen, die damaligen Forstgesetzgebungen.

Die erste Waldbereitung, angeordnet durch Kaiser Maximilian I. im Jahr 1499, hatte das Ziel der Sicherung des Holzbedarfs für die Eisenindustrie am Erzberg (HAFNER 1979; Illmaier 1992). Betroffen waren alle Haupt- und Nebentäler rund um den Erzberg, wobei die bringbaren Bestände verzeichnet wurden. Im Jahr 1501 kam es zur nächsten Waldbereitung, diesmal in den Wäldern des oberen Ennstales. Dies geschah schon in Vorausschau auf den geplanten Rechenbau in Hieflau.

Im Jahr 1524 kam es dann bereits zur zweiten Bereitung in unmittelbarer Umgebung des Erzberges. Es wurden jene Waldungen begutachtet, die im Zuge der ersten Waldbereitung für den Erzberg geschont wurden.

Zwischen 1561 und 1562 fand eine neuerliche Waldbereitung statt, welche alle Hoch- und Schwarzwälder (von Ansiedlungen entfernte sowie von bäuerlicher Wirtschaft unbeeinflusste Wälder) umfasste, die für die Versorgung von Innerberg und Vordernberg in Frage kamen. Aufgenommen wurden Holzvorräte und Kosten der Bringung unter Angabe der erforderlichen Bringungsbauten, sowie Waldschäden. 
Im Jahr 1562 wurde auch das Vieh im Einzugsgebiet der Innerberger Montanindustrie gezählt. Es wurden Rinder und Ziegen verzeichnet, die man auf ausgehackten Beständen, Almen oder bei Schwaighütten antraf.

Im Jahr 1567 fand dann die erste Waldbeschau im Salzatal statt, die ,... Beschaw beschreibung unnd Überschlag der Wäld so dem fuergenomben unnd beratschlagten Rechen geen Reifling auf dem wasser der Salzach bracht mügen ...“ (HAFNER 1979, S. 111) - jene Beschreibung, die für den Bau des Großreiflinger Rechens durchgeführt wurde. Weitere Bereitungen folgten in den Jahren 1596 und 1625 für das Vordernberger Einzugsgebiet (HAFNER 1979).

Die Generalwaldbeschau wurde von 1627 bis 1628 auf Anordnung Kaiser Ferdinand II. durchgeführt. Mit dieser Waldbereitung reagierte man auf den aufkommenden Holzmangel am Erzberg und bei der Salzgewinnung in Aussee. Außerdem fiel die Bereitung mit der Gründung der Innerberger Hauptgewerkschaft zusammen. Die Wälder wurden getrennt nach ihrer Bringbarkeit nach Vordernberg, Innerberg und ins Ausseer Salzkammergut aufgenommen, wobei auch alle Waldschäden aufgezählt wurden. Nach der umfassenden Generalwaldbeschau folgten im Jahr 1682 eine Beschreibung der den Radwerken Vordernbergs gewidmeten Wälder und zwischen 1730 und 1733 die Waldbereitung unter Kaiser Karl VI., die aber durch ,widrige Zufälle in das Steckhen gerathen“ ist und nicht zu Ende geführt wurde (HAFNER 1979).

Nachdem die letzte kaiserlich angeordnete Waldbereitung nicht zu Ende geführt worden war, ordnete Kaiserin Maria Theresia die „General-, Wald-, Bereit-, Berain- und Schätzungskommission“ an. Die in 28 Bänden (Band = lat. tomus, deshalb „Waldtomus“) gedruckten Ergebnisse dokumentieren die umfassendste und detaillierteste historische Waldbereitung. Alle Waldparzellen der Obersteiermark wurden vollständig erfasst, genau lokalisiert und es wurde die erlaubte Nutzung durch den Besitzer festgelegt (HAFNER 1979; NATHER 1964). Mit dem Waldtomus wurden auch erstmals die genauen „Einforstungen“ festgehalten, das heißt es wurden die Namen der Berechtigten und die Anzahl an Weidevieh, das auf bestimmte Parzellen getrieben werden durfte, niedergeschrieben (HasitschKa 2005). Für die Waldbestände führte man eine Unterteilung in vier Altersstufen ein: „wirkmäßiges“ Holz, das sofort schlagbar ist; „, halbgewachsenes“, das in etwa 30 bis 50 Jahren schlagbar wird; ,junge Beschüttung“, in 50 bis 70 Jahren schlagbar, und schließlich „blosser Schlag“, der in 100 bis 150 Jahren zu schlagen sein wird. Die Schätzungen erfolgten in Vordernberger Faß Kohle, die aus den Beständen produziert werden können $\left(1\right.$ Vordernberger Faß $\left.=0,246 \mathrm{~m}^{3}\right)\left(\mathrm{HAFNER}_{\mathrm{A}}\right.$ 1979; HASITSChKa 2005; NATHER 1964).

Das Ergebnis dieser Waldbereitung von 1754 bis 1762 ist in Tabelle 1 zusammengefasst. Die Waldbereitung umfasste 998 Hauptriede mit 20.536 Waldparzellen. In Summe wurde ein Kohlenvorrat von rund 208 Millionen Vordernberger Faß ermittelt, wovon knapp die Hälfte wirkmäßig schlagbar gewesen wäre. Weitere $20,7 \%$ wurden als in 30 bis 50 Jahren schlagbar eingereiht und 17,2\% als junge Beschüttung, die in 50 bis 70 Jahren hiebsreif wird. 15,7\% der aufgenommenen Waldflächen wurden kürzlich abgestockt und standen kahl.

Im Jahr 1787 folgte mit der Einführung des Josephinischen Katasters ein Steueroperat, das den Geldwert der Wälder angab. Das Lagerbuch musste in jeder Steuer- 


\begin{tabular}{|c|c|c|c|c|}
\hline \multicolumn{5}{|c|}{ Gesamtvorrat von 207.773.875 Vordernberger Faß in 998 Hauptrieden } \\
mit 20.536 Waldteilen \\
\hline $\begin{array}{c}\text { davon ent- } \\
\text { fielen auf }\end{array}$ & $\begin{array}{c}\text { wirkmäßiges } \\
\text { (sofort schlagbar) }\end{array}$ & $\begin{array}{c}\text { in 30-50 Jahren } \\
\text { schlagbar }\end{array}$ & $\begin{array}{c}\text { in 50-70 Jahren } \\
\text { schlagbar }\end{array}$ & $\begin{array}{c}\text { in 100-150 Jahren } \\
\text { schlagbar }\end{array}$ \\
\hline $\begin{array}{l}\text { in Vordern- } \\
\text { berger Faß } \\
\text { Holzkohle }\end{array}$ & $96,414.075$ & $42,978.350$ & $35,695.150$ & $32,686.300$ \\
\hline in Prozent & 46,4 & 20,7 & 17,2 & 15,7 \\
\hline
\end{tabular}

Tab. 1: Zusammenfassung der Ergebnisse des Waldtomus (Menhardt 1754-1762, eigener Entwurf)

gemeinde geführt werden und danach hatte jeder Grundbesitzer gemäß der Größe und des Wertes seiner Flächen Steuern zu entrichten. Zugleich mit dem Josephinischen Kataster, der erstmals die Bonität der Waldungen angab, wurde auch die Josephinische Landesaufnahme erstellt, die als erstes genaues topographisches Kartenwerk der Habsburgermonarchie gilt. Die Waldflächen wurden allerdings mit einer einheitlichen Signatur dargestellt, welche keine Rückschlüsse auf den Zustand des Bestandes erlaubt.

Zu Beginn des 19. Jhs. folgten mit dem Franziszeischen Kataster und der Franziszeischen Landesaufnahme die erste genaue Vermessung der Parzellen und das erste Triangulationsnetz des Reiches. Zur Erfassung der Steuerlast wurde ein GrundparzellenProtokoll angelegt, das die einzelnen Waldparzellen oft mit differenziert geschätzten Bonitäten anführte. Das erste, beispielhaft moderne Forsteinrichtungsprojekt wurde im Jahr 1877 begonnen. Aus den Vermessungen und Aufzeichnungen dieser Forsteinrichtung gingen die ersten Bestandskarten der Steiermark hervor, die den Waldbestand je nach Alter des Bewuchses und der Betriebsklasse farblich differenzierten (HASITSCHKA 2005).

\subsection{Auswertung historischer Angaben zur Eisenproduktion am Erzberg}

Weil die Energiequelle Holzkohle mit der Produktion und Weiterverarbeitung von Eisen in engem Zusammenhang steht, können Eisenproduktionsmengen zur Abschätzung der Waldnutzungsintensität in einer Region herangezogen werden.

Eine Abschätzung der Erzförderung am Steirischen Erzberg seit Beginn der Bergbauaktivitäten und für fünf Perioden führte Thalmann (1973) durch. In Tabelle 2 sind die Daten dieser Abschätzung zusammengefasst und die Durchschnittswerte pro Jahr der jeweiligen Periode berechnet. Zwar gab es innerhalb dieser Zeitspannen immer wieder Innovationen, die den Eisenausstoß sprunghaft ansteigen ließen - wie etwa die Industrialisierung des Eisenwesens um das Jahr 1250 oder die Einführung des Floßofens im Jahr 1790. Dennoch zeigt diese Zusammenstellung die enorme Produktionsentwicklung am Erzberg. 


\begin{tabular}{|l|c|c|c|c|c|}
\hline Zeitraum & $\mathbf{3 0 0 - 1 6 0 0}$ & $\mathbf{1 6 0 1 - 1 8 8 0}$ & $\mathbf{1 8 8 1 - 1 9 4 0}$ & $\mathbf{1 9 4 1 - 1 9 6 9}$ & $\mathbf{1 9 7 0 - 1 9 7 2}$ \\
\hline $\begin{array}{l}\text { Anzahl } \\
\text { der Jahre }\end{array}$ & 1.300 & 279 & 59 & 28 & 2 \\
\hline $\begin{array}{l}\text { Produktion } \\
\text { (in t) }\end{array}$ & $1,000.000$ & $14,693.000$ & $64,515.430$ & $71,484.170$ & $10,880.000$ \\
\hline $\begin{array}{l}\text { Produktion } \\
\text { pro Jahr (in t) }\end{array}$ & 769 & 52.663 & $1,093.482$ & $2,553.006$ & $5,440.000$ \\
\hline
\end{tabular}

Tab. 2: Berechnungen aus der geschätzten Erzförderung am Erzberg (THALmanN 1973, eigener Entwurf)

Auf Jahresbasis quantifizierbare Daten finden sich wiederum in alten Wirtschaftsbüchern, beispielsweise in jenen der Innerberger Hauptgewerkschaft, die im Jahr 1625 aus der durch die Regierung angeordnete Fusion der drei Glieder des Eisenwesens, der Innerberger Radmeister (Roheisengewinnung), der von diesen belieferten Hammermeister (Weiterverarbeitung) sowie der Steyrer Eisenhändler oder , Verleger ‘ (Verkauf, Finanzierung)“ (KLEMm 2011, S. 19) hervorging. Mit dem Zusammenschluss der Radmeister, der Hammermeister und der Steyrer Eisenverleger entstand eine Produktions- und Absatzgemeinschaft (IlLMAIER 1992), die den größten Betrieb in Österreich und gleichzeitig eines der größten Bergbauunternehmen der damaligen Welt darstellte (Illmaier 1992; Klemm 2011). In Abbildung 3 ist die Entwicklung der Eisenproduktion der Innerberger Hauptgewerkschaft seit ihrer Gründung bis zum Jahr $1783 \mathrm{zu}$ sehen. Die Werte nach SANDGRUber (1971) sind in q angegeben (1 q = 1 „Pfundzentner“ $=56$ kg) $($ Oppl 1992; SANDGruber 1971).

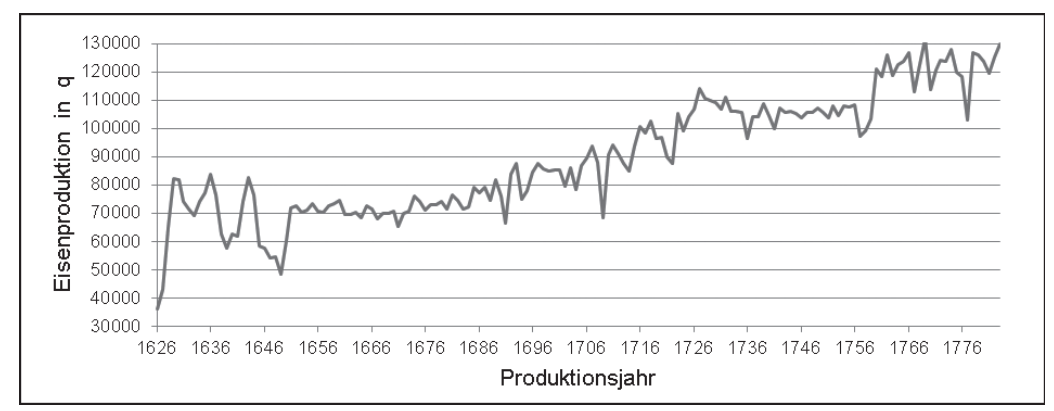

Abb. 3: Graphische Darstellung der Innerberger Eisenproduktion zwischen 1626 und 1783 (S ANDGRUBER 1971, eigener Entwurf)

Neben der produzierten Menge an Roheisen wurden seitens der eisenverarbeitenden Betriebe auch Regien aufgezeichnet, wie etwa jene Mittel, die zum Erwerb von Holzkohle ausgegeben wurden. So geht beispielsweise aus der Zusammenstellung der wöchentlichen Ausgaben eines Innerberger Schmelzwerkes aus dem Jahr 1599 dessen wöchentlicher Bedarf an Holzkohle hervor, der mit 480 Radmeisterfassl (1 Innerberger $\mathrm{Faß}=0,307 \mathrm{~m}^{3}$ ) beziffert wird (PIRCHEGGER et al. 1939). 
In Pirchegger et al. (1939) findet sich ebenfalls ein Hinweis auf den jährlichen Holzkohlenbedarf der gesamten Montanindustrie von Innerberg. Der geschätzte Holzkohlenbedarf ist für die Jahre 1625, 1649 und 1713 mit 300.000 Innerberger Faß Kohle für die Röstanlagen, die zehn Schmelzöfen, einen Hammer und zwei Schmieden in Innerberg angegeben. Das produzierte Eisen der zehn Schmelzöfen versorgte wiederum 60 Hämmer, deren jährlicher Holzkohlenbedarf mit 280.000 Innerberger Faß beziffert wurde (PIRCHEGGER et al. 1939).

ILLMAIER (1992) versuchte den jährlichen Holzkohlenbedarf der gesamten steirischen Eisenindustrie zu erfassen und berechnete für das Jahr 1857 einen Bedarf von 1,1 Mio. $\mathrm{m}^{3}$, was bei einer forstgerechten Nutzung einer Waldfläche von rund 300.000 Hektar entsprechen würde (ILLMAIER 1992).

\subsection{Palynologische Untersuchungen von Moorprofilen}

Eine weitere Methode, die auf die historische Waldnutzung schließen lässt, ist die Pollenanalyse. Durch Siedlungsaktivitäten, Ackerbau und Viehweide reduziert sich die Waldfläche, was eine Verringerung des Niederschlags von Baumpollen sowie eine gleichzeitige Erhöhung des Gras- und Kräuterpollenanteils zur Folge hat (DrESCHERSCHNEIDER 2003). So wird z.B. „,das häufigere Auftreten des Adlerfarnes ... einerseits als Hinweis auf Waldweide ..., andererseits als Zeichen für Brandrodung ... gewertet“" (Drescher-SCHNEIDER 2003, S. 187).

„Die Region um Eisenerz dürfte eine der wenigen Gelegenheiten in den östlichen Ostalpen sein, wo in einem über Jahrtausende besiedelten, aber räumlich abgegrenzten Gebiet hervorragende Ablagerungen für vegetations- und siedlungsgeschichtliche Studien zur Verfügung stehen." (DreScher-SCHNEIDER 2003, S. 191) Einige pollenanalytische Untersuchungsergebnisse liegen auch bereits vor, zum Beispiel vom Moor am Neuburgsattel (MARShAll 1994), vom Profil Griesangermoor (KrAL 1982), vom Oberen Moor auf der Sulzkaralm (DRESCHER-SCHNEIDER 2007) und vom Leopoldsteiner See (Drescher-SCHNEIDER 2003).

\section{Geomorphologische Folgewirkungen von Eingriffen in die Walddecke}

Die Veränderung der Vegetationsdecke im Zuge der über die Jahrhunderte stetig gestiegenen Holzkohlenproduktion hatte umfassende Auswirkungen auf die hydrologischen Verhältnisse an den Standorten. Durch den Wegfall des schützenden Baumbestandes verstärkten sich Oberflächenabfluss und Erosionsprozesse an den genutzten Hängen. Als besonders kritisch sind dabei die Auswirkungen der Brandwirtschaft und des Kahlschlagbetriebes zu sehen. Phasen der Erosionsberuhigung ergaben sich erst wieder durch eine Regeneration der Waldbestände. Im Holzliefergebiet des Steirischen Erzbergs ist somit eine massive mittelalterliche Sedimentmobilisierung zu erwarten, die aber bislang von der österreichischen Geomorphologie noch nicht untersucht wurde. 


\subsection{Thematisierung von geomorphologischen Folgewirkungen in der älteren Literatur über die Erzbergwälder}

Grundsätzlich reichen Einträge mit dieser Thematik nicht weit zurück, denn ,an eine Erhaltung des Waldes als Landschaftselement dachte man (damals weitgehend) nicht. Die zahlreichen Muren und Hochwässer, die nach schweren Gewittern katastrophenartige Ausmaße annahmen, brachte man ... mit der Entwaldung noch nicht in Verbindung. Man fürchtete nur die Holznot, aber nicht die Verkahlung der Hänge und ihre Folgen." (NATHER 1964, S. 182f.) Am wichtigsten war es die Eisenproduktion aufrechtzuerhalten. Das Bewusstsein eines Zusammenhangs zwischen Entwaldung und stärkeren sowie häufigeren Naturkatastrophen war damals noch nicht vorhanden. Naturkatastrophen wurden in ohnmächtiger Abhängigkeit allen irdischen Lebens von höheren Mächten hingenommen (RoHR 2007).

Über die Hochwälder und deren Bewirtschaftung schreibt HцubeK $(1844$, S . 5) beispielsweise, dass diese schlagweise gänzlich kahl abgetrieben und „der unmittelbaren Atmosphäre" ausgesetzt wurden. Durch mangelhafte Vorsorge blieb der natürliche Anflug auf diesen Flächen über Jahrzehnte aus. ,... wegen der Verwilderung der kahlen (Schläge wurde) ... den Winden die Thore in die (benachbarten) Wälder geöffnet, und auf diese Weise jener traurige Zustand herbeigeführt, welchen wir so häufig in den Wäldern der Alpenländer wahrnehmen“. Weiters wurde „,... die Erde bei größerer Elevation abgeschwemmt, und kahle Felsen zu Tage gefördert, bei welchen Jahrhunderte erfordert werden, bis durch den Verwitterungsprozess die Bedingungen einer neuen Vegetation herbeigeführt werden." (HLubeK 1844, S. 5) Als Beispielsfall werden von Hцuвeк (1844) die Wälder in der Umgebung der Kleinalpe (heute Gleinalpe) genannt, wo die geschilderten Folgen des Kahlschlags ,in ihrem ganzen Umfange“ ersichtlich seien (HlubeK 1844, S. 6). Für die Wälder rund um die Kleinalpe 1860 beschreibt er, dass der „... Mangel an Sorgfalt für die Wiederaufforstung der kahlen Schläge die atmosphärischen Niederschläge die Erde von den Bergen ..." abschwemmen lässt (Hlubek 1844; HlubeK 1846, S. 161).

Auch Illmaier (1992) beschreibt eine Nutzungsauswirkung. Im Jahr 1404 kam es in den Tälern rund um den Erzberg zu einem gewaltigen Hochwasserereignis, welches „.... nicht zuletzt auf die großen Überschlägerungen zurückzuführen ..." sei (ILLMAIER 1992, S. 137).

\subsection{Generelle Auswirkungen der Brandwirtschaft}

Waldbrände verursachen grundlegende Veränderungen der Bodenoberfläche sowie der geomorphologischen Aktivität. Es können Verwitterungserscheinungen direkt am anstehenden Gestein hervorgerufen werden. Zusätzlich werden die bodennahe Vegetation sowie die Streuauflage entfernt. Doch auch die einzelnen Bodenhorizonte können bei ausreichend hoher und langer Hitzeeinwirkung in Mitleidenschaft gezogen werden, was eine stark verminderte Infiltrationsrate zur Folge hat. Der daraus resultierende oberflächliche Abfluss, der in verbrannten Wäldern üblich ist, kann erstens die leicht mobilisierbaren Brandrückstände und zweitens die verbrannten Bodenschichten angreifen und erodieren (LoAiciga et al. 2001; Shakesby \& Doerr 2006, u.a.). 
Nach dem Brand herrscht eine erhöhte geomorphologische Aktivität, vor allem durch die stark ansteigende Wassererosion (DIECKMANn et al. 1992; InBAR et al. 1998, u.a.). Eine erhöhte Erosionsrate tritt direkt nach dem Brandereignis ein und dauert, je nachdem wie lang die Vegetationsdecke zur Regeneration benötigt, bis zu zwei Jahre (SHAKESBy \& DoERR 2006). Aber auch seichtgründige Rutschungen können durch einen Waldbrand ausgelöst werden, insbesondere wenn die vormals stabilisierend wirkenden Wurzelsysteme der Bäume und Sträucher absterben (Gray \& Megahan 1981; Meyer \& Pierce 2003, u.a.).

Sото et al. (1995) erforschten die Auswirkungen der Brandwirtschaft auf Boden und Erosion, indem sie drei Versuchsflächen anlegten, wovon eine ursprünglich belassen wurde und zwei mit den üblichen Methoden der Brandwirtschaft bearbeitet wurden. Nach Schlägern und Verbrennen wurde auf den beiden Brandwirtschaftsflächen das gängige Brandkorn Roggen gepflanzt. Bei einem Gesamtniederschlag von 1.636 mm im Versuchszeitraum von neun Monaten betrug der oberflächliche Abfluss der Brandflächen $142 \mathrm{~mm}$ und $181 \mathrm{~mm}$, jene der ursprünglichen Fläche $46 \mathrm{~mm}$. Die gesamte Erosion über die beobachteten neun Monate belief sich bei der belassenen Fläche auf 1,4 Tonnen pro Hektar. Bei den beiden Brandflächen stieg die Erosion auf 45,7 bzw. 57,2 Tonnen pro Hektar an (Soтo et al. 1995).

In der Steiermark ist Brandwirtschaft, d.h. das Abbrennen von geschlagenen Wäldern und die darauf folgende ein- bis zweijährige Aussaat von Getreide aus den Bezirken Bruck an der Mur, Graz, Voitsberg, Weiz, Hartberg, Deutschlandsberg, Leoben und Judenburg überliefert. Brandgrundstücke waren im alten Kataster mit einer eigenen Kulturgattung „Brände“ versehen, deren Gesamtfläche 87.297 Hektar betrug. Mit Einführung des reambulierten Katasters wurden die Brände je nach damaliger Bestockung als Hutweiden oder Wälder ausgewiesen (HAFNER 1979).

\subsection{Generelle Auswirkungen des Kahlschlagbetriebs}

Oberflächlicher Abfluss bestimmt die fluviale Abtragung eines Hanges, die Grundwassersituation wiederum beeinflusst die Hangstabilität. Daher hat jede waldnutzungsbedingte Veränderung der hydrologischen Verhältnisse Auswirkungen auf die geomorphologischen Prozesse. Kahlschläge und das Anlegen von Forststraßen erhöhen die Wassermengen, die direkt auf den Boden auftreffen, was zur Intensivierung der Erosions- und Rutschungsprozesse führt (SLAYMAKer 2000; Wolter et al. 2010, u.a.).

Die Auswirkungen von Waldnutzung auf geomorphologische Prozesse wurden besonders in British Columbia, Kanada, untersucht, wo industrieller Großkahlschlag ohne Maßnahmen der Wiederaufforstung bis ins späte 20. Jh. hinein praktiziert wurde. Durchschnittswerte ergaben eine um das Zehnfache angestiegene Sedimentaustragsrate durch das Einsetzen der Nutzung der Wälder. Außerdem werden die Abflussmengen sowie das zeitliche Auftreten und die Höhe von Überflutungen beeinflusst, indem sich Magnitude und Frequenz von Hochwasserereignissen erhöhen (SLAYMAKER 2000).

Die Untersuchungen zu den Auswirkungen der Waldwirtschaft auf Hanginstabilitäten von GUTHERIE (2002) ergaben nach der Analyse von 363 Rutschungen in drei Einzugsgebieten auf Vancouver Island einen bis zu 24-fachen Anstieg der Rutschungen auf den genutzten Hängen (Gutherie 2002). Eine andere Studie, die sich mit 1.004 
Rutschungen ebenfalls auf Vancouver Island befasste, ergab eine neunfach höhere Rutschungsfrequenz auf den genutzten Hängen gegenüber ungestörten Gebieten. Außerdem traten an den abgeforsteten Hängen auch Rutschungen auf, die unter ungestörten Bedingungen aufgrund ihrer zu geringen Hangneigung nicht ausgelöst worden wären (JАKOB 2000).

WOLTER et al. (2010) bestätigten mit ihrer Untersuchung, dass bei genutzten Hängen geringere Hangneigungen genügen, um Rutschungen auszulösen. Zudem berechneten auch sie eine neunfach höhere Auftrittsrate und eine 3,1-fach vergrößerte von Rutschungen betroffene Fläche an kahlgeschlagenen Hängen gegenüber ungestörten Hängen (WoLTER et al. 2010).

Wie stark die Kahlschlagwirtschaft im Erzberggebiet verbreitet war, verdeutlicht eine Aufstellung der Staatswälder aus dem Jahr 1884. Demnach wurden von den 34.378 Hektar obersteirischen Waldes, der sich damals in Staatsbesitz befand, 26.408 Hektar als Kahlschläge betrieben, das sind beinahe $77 \%$. Etwa $13 \%$ standen unter normalem Plenterbetrieb und die restlichen rund $10 \%$ im Plenterbetrieb als Schutzwald (HAFNER 1979).

\subsection{Folgewirkungen der Regeneration von genutzten Waldungen}

Die Auswirkungen von Aufforstungen und natürlicher Wiederbewaldung von Einzugsgebieten auf die Erosion und den Sedimentaustrag wurden beispielsweise im oberen Teil des Drôme-Flusses in Südfrankreich untersucht. Im Jahr 1835 waren rund $17 \%$ des Einzugsgebietes bewaldet. Nach der Aufgabe zahlreicher landwirtschaftlich und forstwirtschaftlich genutzter Flächen zu Beginn des 20. Jhs. waren im Jahr 1928 bereits 33\% und im Jahr $195436 \%$ mit Wäldern bedeckt. Nach einem starken Rückgang der Beweidung stieg die Waldfläche im Untersuchungsgebiet auf 54\% im Jahr 1970 und auf 55\% im Jahr 1988. In den Sedimentationsgebieten zeigte sich ein deutlicher Rückgang der Sedimentationsraten seit dem Jahr 1835. Außerdem verringerten sich der gesamte durchschnittliche Abfluss des Drôme-Flusses sowie das durchschnittliche Volumen von Überflutungen (PIEGAY et al. 2004).

Sснміт et al. (2003) stellten für ein kleines Einzugsgebiet in Nordbayern eine Zunahme der Erosion durch Abholzungen seit dem Mittelalter fest, auf welche ebenfalls eine Abnahme des Sedimentaustrages seit der Mitte des 19. Jhs. folgte. Der Rückgang wird mit der damals einsetzenden Wiederbewaldung, nicht zuletzt wegen der Aufgabe von degradierten landwirtschaftlichen Flächen, erklärt. Gegenwärtig ist die Erosionsrate in diesem Gebiet durch die beinahe gänzlich geschlossene Walddecke sehr gering (Sснмітт et al. 2003).

Eine Quantifizierung der Auswirkungen einer Aufforstung führten ZHENG et al. (2008) auf Versuchsflächen durch. Aufgeforstete Flächen wiesen dabei innerhalb von vier Jahren einen Rückgang des Oberflächenabflusses um 63,0 bis $88,1 \%$ sowie einen Rückgang der Bodenerosion um 75,5 bis 97,1\% gegenüber den nicht aufgeforsteten Flächen auf (Zheng et al. 2008).

Die Waldungen im Einzugsgebiet der historischen Holzkohlenproduktion rund um den Erzberg wurden speziell mit dem Einsetzen des Großkahlschlagumtriebes seit dem 
13. Jh. in einem wiederkehrenden Zyklus von Abholzung und natürlicher Regeneration geführt. Die Dauer der Regenerationsphasen hing dabei stark von der Beschüttungszeit ab, die die jeweiligen Standorte für die neuerliche Bildung eines Waldbestandes benötigten. Diese wiederum wurde durch eine auf den Kahlschlag folgende Nutzung verlängert. „Die ausgedehnten Kahlschläge der großen Forste werden, kaum geräumt, der Weide geöffnet; noch nie ist ein obersteirischer Holzschlag in Hege gelegt worden." (Wessely 1853b, S. 55) Der Druck durch das Weidevieh hemmte den natürlichen Anflug und verhinderte ein Aufkommen des Waldes über Jahre bis Jahrzehnte (HAFNER 1979; Wessely 1853a; Wessely 1853b).

\subsection{Gebietsspezifische Befunde aus Bodenaufschlüssen und Bohrungen}

Durch die intensive Nutzung der obersteirischen Wälder für die Holzkohlenproduktion erhöhten sich die Erosionsraten an den genutzten Hängen sowie die Sedimentationsraten in den Akkumulationsbereichen, am Hangfuß, in Mulden, in Au- und Talbereichen, an Talausgängen usw. In den abgelagerten Schichten der entsprechenden Sedimentspeicher müssen die Folgen der Nutzungsaktivitäten sichtbar werden. Mit der Anlage von Bodenaufschlüssen und Bohrlöchern an geeigneten Positionen innerhalb der Ablagerungsbereiche erhält man ein tiefer gehendes Verständnis über die Nutzungsgeschichte des dahinter liegenden Einzugsgebietes.

Als Beispiel soll ein ursprünglich von Nicola RAMPAZzo (Institut für Bodenforschung, Universität für Bodenkultur, Wien) dokumentierter Bodenaufschluss dienen, dessen Existenz und Position den Autoren freundlicherweise mündlich mitgeteilt wurde. Der Aufschluss, der sich auf einem Ablagerungsbereich am Ausgang eines kleinen Einzugsgebietes im Jassinggraben nordwestlich von Tragöß befindet, ist in Abbildung 4 zu sehen. In der Karte links wurde der Standort des Bodenprofils im nördlichen Bereich des gut zu erkennenden Schwemmfächers mit einem Kreis markiert. Das dahinter liegende Einzugsgebiet wurde innerhalb der oberirdischen Wasserscheide grau markiert. Das Gebiet des Kartenausschnitts befindet sich in einer Entfernung von etwa zehn Kilometern Luftlinie östlich des Erzberges. In der Photographie rechts sind die Horizontmächtigkeiten des carbonathaltigen Schwemmbodens angegeben.

Sofort ins Auge fällt der mächtige Kohlenhorizont (Cu1 in Abb. 4), dessen Herkunft nicht eindeutig geklärt ist. Grundsätzlich kann sich die Kohlenschicht aus eingeschwemmten Rückständen historischer Kohlplätze im Einzugsgebiet zusammensetzen, was eventuell nur auf den obersten Teil der Schicht zutrifft, der mit Sediment durchsetzt zu sein scheint. Beim unteren Bereich spricht die außerordentliche Mächtigkeit und Einheitlichkeit des Kohlenhorizonts dafür, dass das Bodenprofil am Standort einer alten Verkohlungsstätte angelegt wurde. Aus montanwirtschaftlicher und geomorphologischer Sicht wäre beides durchaus möglich. Dies könnte der Fall sein, wenn zunächst die vom Tal aus leicht zu erreichenden Wälder des Schwemmfächers verkohlt wurden und man später mit den Kohlenmeilern in höhere Lagen des Einzugsgebietes nachrückte. Außerdem sind die darüber liegenden Sedimentschichten gut zu erkennen, welche die Kohlenschichten später überlagerten. 

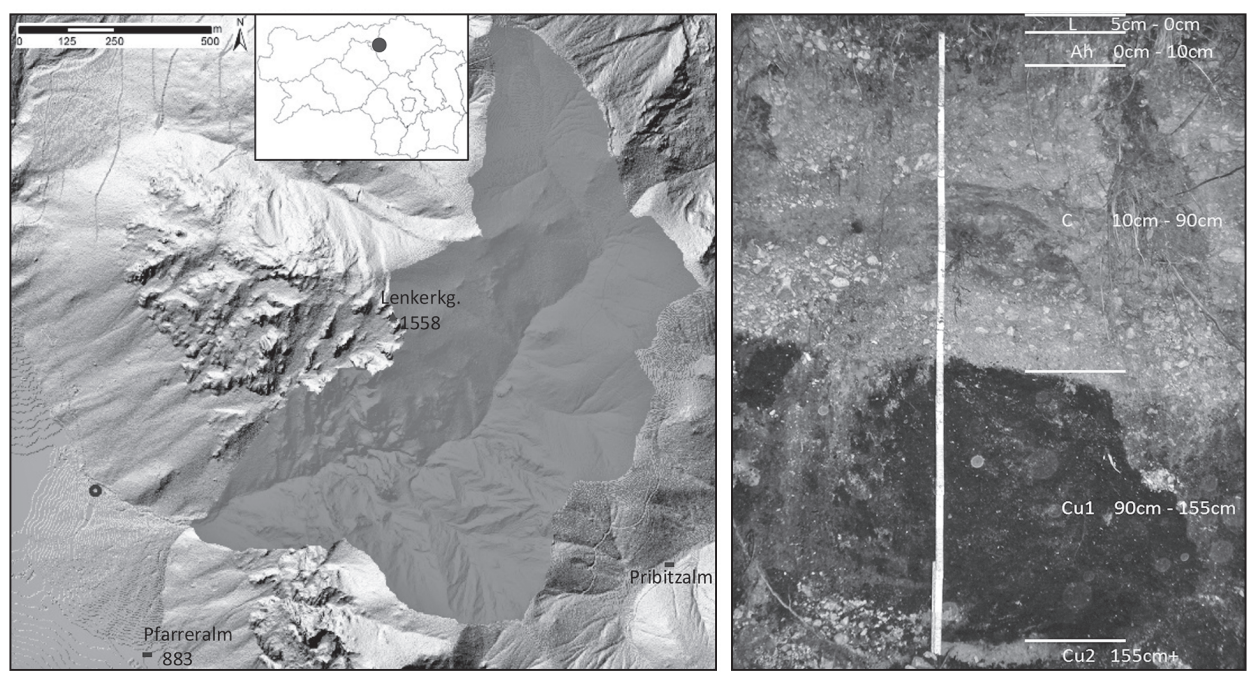

Abb. 4: Links: Standort des Bodenprofils (GIS-Steiermark). Rechts: Photographie des Bodenprofils (eigene Aufnahme)

\section{Conclusio}

Die Nutzung der obersteirischen Wälder war über Jahrhunderte hinweg äußerst intensiv. Besonders ab der Mitte des 13. Jhs. stieg mit der Eisenproduktion der Bedarf an Holzkohle sprunghaft an und wuchs seither immer weiter. Großkahlschläge bestimmten als dominierende Rodungsart das Landschaftsbild und standen in enger Verbindung mit der Errichtung von Bringungsanlagen. Mit den Erbauungsdaten dieser Anlagen ist es möglich einen Zeitpunkt zu fixieren, ab dem die intensive bzw. die erstmalige Nutzung von einzelnen Einzugsgebieten einsetzte. Die Daten der Waldbeschreibungen, die von den Landesfürsten als prospektives Instrument zur Erhebung der Versorgungslage eingesetzt wurden, können zur lokalen Rekonstruktion des Waldbestandes herangezogen werden. Eine grobe Abschätzung des Zustandes der Vegetationsdecke auf regionaler Ebene wird mit der Auswertung der Eisenproduktionsmengen am Erzberg und den verbrauchten Holzkohlenmengen sowie mit palynologischen Untersuchungen erreicht.

Traditionelle Nutzungsformen wie Brandwirtschaft und Großkahlschläge, die in den obersteirischen Wäldern jahrhundertelang praktiziert wurden, trugen erheblich zur Sedimentmobilisierung an den genutzten Hängen bei. Dieser Prozess verlangsamte sich nach einer Nutzungsaufgabe, bei der sich der Waldbestand erholen konnte. Durch das Anlegen und Auswerten von Bodenprofilen und Bohrlöchern auf Sedimentspeichern, etwa bei Talausgängen, können die Nutzungsgeschichte und deren Auswirkungen auf Erosion und Sedimentation im dahinter liegenden Einzugsgebiet rekonstruiert werden. Die Folgen der Waldverwüstungen, die anthropogen induzierte Hangdenudation im Erzberggebiet, werden so quantifizierbar. 
Die umfassenden Eingriffe in die Vegetationsdecke sowie der gleichzeitig durch historische Daten über mehrere hundert Jahre in die Vergangenheit lokal rekonstruierbare Waldzustand prädestinieren das Erzberggebiet geradezu für ein breites Band an Fragestellungen moderner geomorphologischer Forschungsfelder. Einzelne kleine Einzugsgebiete könnten in zukünftigen Untersuchungen modellhaften Charakter für die Erstellung von Sedimentbilanzen oder die Quantifizierung von anthropogen ausgelösten Erosionsprozessen gewinnen.

\section{Literaturverzeichnis}

Agricola G. (1557), Vom Bergkwerck xij Bücher darin alle Empter Instrument Bezeuge... Basel, Froben.

Ast H., Ast W., Katzer E. (1970), Holzkohle und Eisen. Beitrag zur Volkskunde, Wirtschaftsund Sozialgeschichte des Raumes um Gutenstein (= Niederösterreichische Volkskunde, 6). Linz, Rudolf Trauner.

Biringuccio V. (1559), De la pirotechnia. Venetia, P. Gironimo Giglio.

Dieckmann H.H., Motzer H., Harres H.P., Seuffert O. (1992), Vegetation and erosion. Investigations on erosion plots in southern Sardinia. In: Geo-Öko-Plus, 3, S. 139-149.

Drescher-SchneIder R. (2003), Die Vegetations- und Besiedlungsgeschichte der Region Eisenerz auf der Basis pollenanalytischer Untersuchungen im Leopoldsteiner See und in der Eisenerzer Ramsau. In: KLemm S. (Hrsg.), Montanarchäologie in den Eisenerzer Alpen, Steiermark. Archäologische und naturwissenschaftliche Untersuchungen zum prähistorischen Kupferbergbau in der Eisenerzer Ramsau (= Mitteilungen der Prähistorischen Kommission, 50), S. 174-197. Wien, Verlag der Österreichischen Akademie der Wissenschaften.

Drescher-Schneider R. (2007), Spät- und postglaziale Vegetationsentwicklung im Oberen Moor - Sulzkaralm. Bericht Dezember 2007. Kainbach bei Graz, Nationalpark Gesäuse.

Du Hamel du Monceau H.L., Oelhafen von Schöllenbach C.C., Winterschmidt A.W. (1766), Von Fällung der Wälder und gehöriger Anwendung des gefällten Holzes. Oder wie mit dem Schlag-Holz, dann halb- und ganz ausgewachsenem Ober-Holz, umzugehen, und alles bekannte Holz richtig zu schätzen und anzuschlagen ist. Nürnberg, A.W. Winterschmidt.

Gray D.H., Megahan W.F. (1981), Forest vegetation removal and slope stability in the Idaho batholith. Intermountain Forest and Range Experimental Station (= Research Paper, INT, 271). Ogden, Utah, United States Department of Agriculture Forest Service.

Gutherie R.H. (2002), The effects of logging on frequency and distribution of landslides in three watersheds on Vancouver Island, British Columbia. In: Geomorphology, 43, S. 273-292.

Hafner F. (1979), Steiermarks Wald in Geschichte und Gegenwart - Eine forstliche Monographie. Wien, Österreichischer Agrarverlag.

HasitschKa J. (2005), Gesäusewälder - Eine Forstgeschichte nach Quellen von den Anfängen bis 1900 (= Schriften des Nationalparks Gesäuse, 1). Gröbming, Wallig.

Hцubek F.X. (1844), Betrachtungen über die Bewirthschaftung der Hochwälder in ihrer Anwendung auf die Obersteiermark. Ein Vortrag für die XXVI. allgemeine Versammlung der k.k. steiermärkischen Landwirthschafts-Gesellschaft. Graz, Verlag von den A. Leykam“schen Erben.

Hlubek F.X. (1846), Die Landwirthschaft des Herzogthumes Steiermark. Festgabe für die Mitglieder der X. Versammlung deutscher Land- und Forstwirthe. Graz, J. A. Kienreich. 
Illmaier F. (1992), Der steirische Erzberg in Verknüpfung mit dem steirischen Wald in Vergangenheit und Gegenwart. In: Manfreda A.K., Sika P. (Hrsg.), Aus der Geschichte des Erzbergbaues im zentraleuropäischen Raum. Vorträge des 4. Erzberg-Symposiums in Eisenerz 19.-22. Oktober 1988 (= Leobner Grüne Hefte, 10). S. 135-146. Wien, Montanhistorischer Verein für Österreich, Leoben.

Inbar M., TAMir M., Wittenberg L. (1998), Runoff and erosion processes after a forest fire in Mount Carmel, a Mediterranean area. In: Geomorphology, 24, S. 17-33.

Јаков М. (2000), The impacts of logging on landslide activity at Clayoquot Sound, British Columbia. In: Catena, 38, S. 279-300.

Killian H. (1994), Holzkohle und Köhlerei. Ein forsthistorischer Beitrag zu einem alten Waldgewerbe. In: Ast H., Wernhart K.R. (Hrsg.), Köhlerei in Europa und der Karibik, Kulturanthropologische Studien basierend auf einem Symposium im Waldbauernmuseum Gutenstein (= Wiener Beiträge zur Ethnologie und Anthropologie, 6), S. 15-34. Wien, WUV-Universitätsverlag.

Klemm S. (2003), Montanarchäologie in den Eisenerzer Alpen. In: Klemm S. (Hrsg.), Montanarchäologie in den Eisenerzer Alpen, Steiermark. Archäologische und naturwissenschaftliche Untersuchungen zum prähistorischen Kupferbergbau in der Eisenerzer Ramsau (= Mitteilungen der Prähistorischen Kommission, 50), S. 11-44. Wien, Verlag der Österreichischen Akademie der Wissenschaften.

Klemm S. (2011), Straßen für den Erzberg. Archäologisch-historische Altstraßenforschung in der Steiermark, 16.-18. Jahrhundert. Münster - Wien, LIT-Verlag.

Kral F. (1982), Pollenanalytische Untersuchungen im Schoberpaßgebiet als Beitrag zur postglazialen Waldgeschichte der Steiermark. In: Phyton Austria, 22, S. 243-265.

LeinBöcK J.G. (1834), Die Forstwirthschaft mit Beziehung auf den Bergbau, Erster Theil, WaldAbtrieb und Wald-Cultur. Leipzig, Otto Wigand'sche Verlags-Erpedition.

Loaiciga H.A., Pedreros D., Roberts D. (2001), Wildfire-streamflow interactions in a chaparral watershed. In: Advances in Environmental Research, 5, S. 295-305.

Marshall P.D. (1994), The Environmental Impact of Mining and Metalworking Activities in Steiermark, Austria. Dissertation, University of Sheffield.

MaYrhofer R.J., HAMPL F. (1958), Frühgeschichtliche Bauernrennfeuer im südöstlichen Niederösterreich. Arbeitsbericht über die Grabungen 1950 und 1955 und Naturwissenschaftlichtechnologische Untersuchungen der Grabungsfunde (= Archaeologia Austriaca, Beiheft 2). Wien, Franz Deuticke.

Menhardt G. (1754-1762), General-, Wald-, Bereit-, Berain- und Schätzungs-CommissionsBeschreibung im Erb-Herzogthum Steyer (= Waldtomus, 28 Bände). Steyr, Gregori Menhardt Verlag.

Meyer G.A., Pierce J.L. (2003), Climatic controls on fire-induced sediment pulses in Yellowstone National Park and central Idaho: A long-term perspective. In: Forest Ecology and Management, 178, S. 89-104.

NAtHer H. (1964), Die Waldwirtschaft im Gebiet von Murau (= Schwarzenbergischer Almanach, $34,1968)$. Murau.

Oppl F. (1992), Stadt und Eisen (= Beiträge zur Geschichte der Städte Mitteleuropas, 11). Linz, Der Arbeitskreis.

Piegay H., Walling D.E., Landon N., He Q., Liebault F., Petiot R. (2004), Contemporary changes in sediment yield in an alpine mountain basin due to afforestation (the upper Drôme in France). In: Catena, 55, S. 183-212.

Pirchegger H., von Geramb V., Riehl H. (1939), Steirisches Eisen - Das Steirische Eisenwesen von 1564 bis 1625 (= Beiträge zur Geschichte des ostmärkischen Eisenwesens, 3 ). Graz, Leykam. 
Pömer K., Straub D. (1980), Die Hallstattkultur - Frühform europäischer Einheit (= Festschrift zur internationalen Ausstellung des Landes Oberösterreich, 25. April bis 26. Oktober 1980, Schloß Lamberg, Steyr). Linz, Oberösterreichischer Landesverlag.

RoHr C. (2007), Extreme Naturereignisse im Ostalpenraum. Naturerfahrungen im Spätmittelalter und am Beginn der Neuzeit. Köln et al., Böhlau.

SANDGRuber R. (1971), Der Scheibbser Eisen- und Provianthandel vom 16. bis ins 18. Jahrhundert mit besonderer Berücksichtigung preis- und konjunkturgeschichtlicher Probleme. Dissertation, Universität Wien, Philosophische Fakultät, Institut für Geschichte.

Schlick L., von Giganth P.A.F., von Hassfeld C.F. (1767), Erfrischt- und in etwas vermehrte Waldordnung in Erbherzogthum Steyer. Steyr, Gregori Menhardt.

Schmitt A., Dotterweich M., Schmidtchen G., Bork H.-R. (2003), Vineyards, hopgardens and recent afforestation: Effects of late Holocene land use change on soil erosion in northern Bavaria, Germany. In: Catena, 51, S. 241-254.

Schopf W., Schof F.J. (1843), Die Waldordnung des Landes Steyermark vom 26. Juny 1767, mit den nachgefolgten noch giltigen Vorschriften. Grätz, Kienreich.

Shakesby R.A., Doerr S.H. (2006), Wildfire as a hydrological and geomorphological agent. In: Earth-Science Reviews, 74, S. 269-307.

Slaymaker O. (2000), Research developments in the hydrological sciences in Canada (19951998): Surface water-quantity, quality, and ecology. In: Hydrological Processes, 14, S. 1539-1550.

Soto B., Basanta R., Perez R., Diaz-Rierros F. (1995), An experimental study of the influence of traditional slash-and-burn practices on soil erosion. In: Catena, 24, S. 13-23.

Thalmann F. (1973), Probleme der Abbauplanung und der Qualitätssteuerung am Steirischen Erzberg in Abhängigkeit von den geologisch-mineralogischen Verhältnissen (= Mitteilungen der Geologischen Gesellschaft in Wien, 66, 67). Wien.

Wessely J. (1853a), Die österreichischen Alpenländer und ihre Forste. Erster Theil: Die Natur, das Volk, seine Wirthschaft und die Forste der Oesterreichischen Alpenländer. Wien, Wilhelm Braumüller.

Wessely J. (1853b), Die österreichischen Alpenländer und ihre Forste. Zweiter Theil: Forststatistik der Oesterreichischen Kronlaender Kaernthen, Krain, Salzburg, Steiermark, Tirol und Vorarlberg. Wien, Wilhelm Braumüller.

Wolter A., Ward B., Millard T. (2010), Instability in eight sub-basins of the Chilliwack River Valley, British Columbia, Canada: A comparison of natural and logging-related landslides. In: Geomorphology, 120, S. 123-132.

Zheng H., Chen F., Ouyang Z., Tu N., Xu W., Wang X., Miao H., Li X., Tian Y. (2008), Impacts of reforestation approaches on runoff control in the hilly red soil region of Southern China. In: Journal of Hydrology, 356, S. 174-184. 